\title{
Pediatría
}

http://www.revistapediatria.org/

DOI: https://doi.org/10.14295/rp.v53i1.158

\section{Reporte de Caso}

\section{Reporte de caso y revisión de literatura: Enfermedad de orina con olor a jarabe de arce}

\section{Adolfo Enrique Álvarez Montañez ${ }^{a}$, Sindy Margareth Bermejo Padilla ${ }^{b}$, Sandra Yadira Stapper Páez $^{\mathrm{C}}$}

a Neuropediatra Clínica General del Norte. Docente de Postgrado Pediatría Universidad Libre y Simón Bolívar, Barranquilla, Colombia. E-mail: adolfoalvarezmd@yahoo.com

b Médico Pediatra, Caja de Compensación familiar CAFAM, Bogotá, Colombia. E-mail: shin_02@hotmail.com

c Residente de Pediatría, Universidad Metropolitana, Barranquilla, Colombia. E-mail: Sandra_stapper@hotmail.com

INFORMACIÓN DEL ARTÍCULO

Historia del artículo:

Recibido el 27 septiembre de 2019

Aceptado el 03 de julio de 2020

Palabras clave:

Errores Innatos del Metabolismo

de los Aminoácidos

Enfermedad de la Orina de Jarabe

de Arce.

Aminoácidos de Cadena Ramificada

Leucina

Isoleucina

Valina.

\section{R E S U M E N}

Antecedentes: la enfermedad de orina con olor a jarabe de arce, por su olor similar a la miel del árbol tipo arce o «azúcar quemada», es un trastorno hereditario de los aminoácidos de cadena ramificada con diferentes expresiones fenotípicas; en periodos agudos genera acumulación de neurotóxicos que requiere un diagnóstico y manejo oportuno por las secuelas neurológicas y desenlace fatal.

Resumen del caso: Se presenta el caso de una recién nacida a término, embarazo controlado, antecedentes negativos. Ingresa al sexto día de vida con irritabilidad, succión deficiente, fiebre subjetiva y emesis. Evolución tórpida con falla ventilatoria, compromiso neurológico y acidosis. Se investiga error innato del metabolismo instaurando manejo inicial. Se recibe reporte de elevación de aminoácidos de cadena ramificada, confirmándose diagnóstico de enfermedad de orina con olor a jarabe de arce.

Conclusiones: con la presentación de este caso clínico se hace una revisión de la literatura, en relación con la fisiopatología y diagnóstico y se muestran las recomendaciones de tratamiento, que a la luz de la evidencia actual favorecen un control metabólico, intentando disminuir la mortalidad y compromiso neurológico a futuro.

\footnotetext{
*Autor para correspondencia. Sindy Margareth Bermejo Padilla
} Correo electrónico: shin_02@hotmail.com

Como Citar: Álvarez A. Bermejo S. Stapper S. Reporte de caso y revisión de literatura: Enfermedad de orina con olor a jarabe de arce. Pediatr. 2020;53(1): 30-35. 


\section{Case report and literature review: Maple syrup urine disease}

A B S T R A C T

\section{Keywords:}

Amino Acid Metabolism

Inborn Errors

Maple syrup urine disease

Amino Acids

Branched-Chain

Leucine

Isoleucine

Valine.
Background: The maple syrup urine disease is known for its smell, which is like honey from the maple tree or like "burned sugar". It is a hereditary disorder of branched-chain amino acids with different phenotypic expressions. The acute period generates an accumulation of neurotoxins requiring early diagnosis and treatment due to neurological sequelae and fatal outcome.

Clinical case: We present the case of a newborn at term pregnancy with an unremarkable story. She was admitted after six days of irritability, poor suction, subjective fever, and emesis. The patient had unfavorable evolution with ventilatory failure, neurological signs, and acidosis. Inborn error of metabolism was suspected, and initial treatment was established in consonance with the clinical suspicion. A report of an increase of branched-chain amino acids confirmed maple syrup urine disease.

Conclusions: Presenting this case, we make a non-systematic review of the literature, including pathophysiology, diagnosis, and treatment guidelines, showing current evidence, for metabolic control, avoiding mortality, and significant neurological outcome.

\section{Introducción}

La enfermedad de orina con olor a jarabe de arce o MSUD, del inglés: maple syrup urine disease (OMIM \#24860; ORPHA :511), es un error innato del metabolismo (EIM) de los aminoácidos de cadena ramificada (AACR). Esta aminoacidopatía, es una enfermedad huérfana con frecuencia mundial de aproximadamente 1 en 185000 (1). En Colombia no se tienen datos de la prevalencia de esta condición. Por lo general, la enfermedad es inicialmente asintomática, mientras que, durante la crisis metabólica, se presenta con clínica de intoxicación e importante compromiso neurológico. Se describe un caso clínico colombiano con revisión de la literatura, fisiopatología, diagnóstico y se muestran recomendaciones de tratamiento.

\section{Caso clínico}

Paciente femenina de seis días de edad que consulta a urgencias por cuadro de 24 horas de evolución de irritabilidad, succión deficiente, fiebre y emesis. Madre de 21 años, G4C3A1 . La paciente es producto de cuarta gestación de padres no consanguíneos. Cesárea a las 40 semanas de edad gestacional, sin complicaciones, peso $3178 \mathrm{~g}$, talla $49 \mathrm{~cm}$, perfil infeccioso negativo y antecedentes familiares negativos. Al examen físico se presenta hipoactiva, hipotónica, no abombamiento de fontanelas, ni olor llamativo. Ingresa a unidad de cuidado intensivo neonatal (UCIN) por sospecha de sepsis neonatal y se inicia antibioticoterapia. Los paraclínicos iniciales indicaron, cetonuria de $150 \mathrm{mg} / \mathrm{dl}$ y glucosa de $150 \mathrm{mg} / \mathrm{dl}$. Los gases indicaron un $\mathrm{pH}$ de 7.09; PCO2 de 56.2 $\mathrm{mmol} / \mathrm{l}$; PO2 de $59.3 \mathrm{mmol} / \mathrm{l}$; HCO3 de $16.7 \mathrm{mmol} / \mathrm{l}$ y base exceso de $-13.3 \mathrm{mmol} / \mathrm{l}$.
Con signos clínicos de encefalopatía neonatal y sin mejoría con el tratamiento, se evidenció la fontanela anterior amplia y la paciente presentó crisis neonatales tónicas en extensión. Fue valorada por neuropediatría y genética, quienes solicitaron amonio y lactato en plasma, obteniendo como resultado niveles de $116 \mu \mathrm{mol} / \mathrm{l}(197.55 \mu \mathrm{g} / \mathrm{dl})$ de amonio y de lactato sérico en $2 \mathrm{mmol} / \mathrm{L}$, lo cuales fueron interpretados como anormales, por encima del límite superior. La ecografía transfontanelar fue reportada como normal, mientras que la tomografía craneal simple informó hipodensidad bilateral, con mala diferenciación entre sustancia gris y blanca. el electroencefalograma evidenció descargas aisladas de punta onda bilaterales generalizadas.

Se sospecha de EIM, solicitándose cuantificación de aminoácidos en plasma por técnica de HPLC (AP-HPLC) y ácidos orgánicos cualitativos en orina (AOU), se inicia dieta libre de proteínas con $130 \mathrm{Kcal} / \mathrm{kg} /$ día por 48 horas y reinicio de proteínas según niveles de amonio en plasma. A los 8 días se recibe reporte de AOU con detección de ácido ceto-isovalerico, ácido ceto-isocaproico y cuerpos cetónicos (elevados marcadamente), metabolitos de los AACR. Se hace diagnóstico de MSUD prescribiéndose manejo con dieta libre de AACR y tiamina a 200 mg/día, sin embargo, dada la evolución clínica ulterior se consideró no respondedora a tiamina. Además, se solicitó inicio de fórmula láctea especial sin AACR.

Al día 10 de vida, persiste deterioro neurológico y crisis neonatales manejadas con fenobarbital, requiriendo soporte ventilatorio invasivo. Al día 20, se recibe reporte de AP-HPLC, demostrando valores elevados de leucina $2348 \mu \mathrm{mol} / \mathrm{l}(48-$ 175), aloisoleucina $120 \mu \mathrm{mol} / \mathrm{l}(<2)$, valina $474 \mu \mathrm{mol} / \mathrm{l}(33-300)$ e isoleucina $262 \mu \mathrm{mol} / \mathrm{l}(31$ - 105). Se considera realizar diálisis peritoneal para remoción de los metabolitos neurotóxicos. Es remitida a institución de cuarto nivel, donde ingresa el día 30 bajo ventilación mecánica, somnolienta y con episodios de opistótonos. El control de amonio evidenció niveles de 278 
$\mu \mathrm{mol} / \mathrm{l}(473.43 \mu \mathrm{g} / \mathrm{dl})$. Se inicia diálisis peritoneal además aporte proteico y calórico controlado. Quince días después, luego de mejoría clínica y normalización de los niveles de amonio, se toman nuevos AOU. Presenta una buena evolución, con funciones neurológicas estables, tolera suspensión del soporte ventilatorio y ajuste del aporte enteral. Se permite el egreso a los pocos días con plan dietario especializado, apoyo psicológico familiar y seguimiento por neurología infantil, rehabilitación para estimulación y valoración por genética. Se recibió reporte posterior a egreso de los últimos AOU solicitados, evidenciando el descenso de leucina en $319 \mu \mathrm{mol} / \mathrm{l}(58$ - 171), isoleucina de $6 \mu \mathrm{mol} / \mathrm{l}(21$ - 90) y de valina en $164 \mu \mathrm{mol} / \mathrm{l}(84-318)$.

\section{Discusión}

La MSUD es un trastorno autosómico recesivo con diversas variantes fenotípicas de presentación clínica y presenta un riesgo de recurrencia del $25 \%$. En algunas poblaciones presenta una prevalencia muy alta, por ejemplo, en los menonitas es de $1: 358$ (2) y en los gallegos (España) de $1: 52,541$ (3). En Colombia no se tienen datos precisos de la prevalencia de esta condición. Gómez-Castro et al., en 2008, presentaron un reporte de caso de MSUD neonatal en Bogotá, con clínica inicial desde el segundo día de vida, similar a la reportada en la literatura y con adecuada respuesta al tratamiento (4). Páez et al., en 2015 reportaron un caso neonatal en Bogotá, en un pretérmino con presentación clínica al quinto día de vida, con cuadro similar al presente paciente, pero con evolución tórpida y posterior fallecimiento (5). En ambos casos reportados, al igual que en el presente caso, no se encontró el olor característico a «Jarabe de Arce»

\section{Fisiopatología}

La enfermedad se origina en la deficiencia del complejo multienzimático deshidrogenasa de los alfacetoácidos ramificados o BCKDH, del inglés: branched-chain alpha-keto acid dehydrogenase complex, el cual es requerido para el metabolismo de los AACR (6), La deficiencia de BCKDH genera la posterior acumulación de AACR y sus metabolitos alfacetoácidos (figura 1). El acumulo de estos metabolitos origina un síndrome de intoxicación, la leucina se comporta como un neurotóxico que produce edema cerebral, natriuresis e hiponatremia secundaria $(7,8)$. Los AACR son cetogénicos y glucogénicos (1) y son liberados ante episodios de catabolismo proteico como el ayuno, estrés por infecciones, vacunas y fiebre, los cuales deben considerarse como factores importantes en el debut de la enfermedad y en el posterior control metabólico. El complejo multienzimático tiene cuatro subunidades, codificadas por tres genes diferentes (DBT, 1p21.2; BCKDHB, 6q14.1 y BCKDHA, 19q13.2), lo que explica sus diferentes fenotipos clínicos $(9,10)$ (tabla 1). Hasta la fecha, más de 200 mutaciones han sido reportadas.

\section{Presentación clínica}

Se han descrito al menos cinco fenotipos clínicos (tabla 2). En los casos de paciente con fenotipos atípicos, intermedios o controlados tempranamente, se presenta una mayor tasa de ansiedad, depresión, trastorno por déficit de atención con hiperactividad, trastornos del movimiento y pequeñas reducciones en inteligencia y función global (11).<smiles>CC(C)C[C@H](N)C(=O)O</smiles>

Leucina

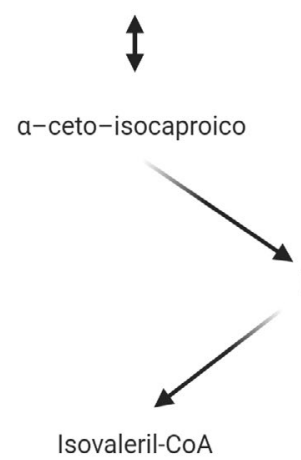<smiles>CC[C@H](C)[C@H](N)C(=O)O</smiles>

Isoleucina

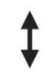

a-ceto-metilvalérico

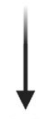

Bloqueo Metabólico

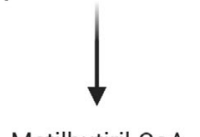

Metilbutiril-CoA<smiles>CC(=O)[C@H](N)C(C)C</smiles>

Valina

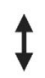

a-ceto-isovalérice

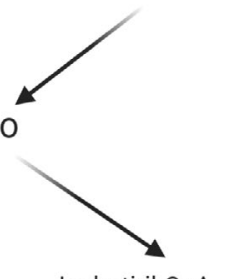

Figura 1. Vía metabólica de los aminoácidos de cadena ramificada. 
Tabla 1. Componentes principales de la enzima alfa- cetoácido deshidrogenasa

\begin{tabular}{|c|c|c|c|}
\hline \multicolumn{2}{|l|}{ Componente } & Gen(locus) & $\%$ patologías \\
\hline \multicolumn{4}{|l|}{ Subunidad E1* } \\
\hline & E1a & BCKDHA $^{* *}(19 q 13.2)$ & $45 \%$ \\
\hline & E1b & BCKDHB (6q14.1) & \\
\hline Subunidad & E 2 & $\mathrm{DBT}^{* * *}(1 \mathrm{p} 21.2)$ & $35 \%$ \\
\hline Subunidad & E $3 \dagger$ & $\mathrm{DLD}+(7 q 31.1)$ & $20 \%$ \\
\hline \multicolumn{4}{|l|}{ Subunidades reguladoras } \\
\hline & nasa & BCKDK (16p11.2) & \\
\hline & tasa & PPM1K (4q22.1) & \\
\hline
\end{tabular}

* Tiamina dependiente **BCKDH: Decarboxilasa de alfa-cetoácido de cadena ramificada;

***DBT: Dihidrolipoil transacilasa; $†$ Se comparte con los complejos de piruvato y alfa-cetoglutarato deshidrogenasa.

††DLD: Lipoamida deshidrogenasa. Adaptado de $(10,11)$

Tabla 2. Fenotipos clínicos de la enfermedad de orina de jarabe de arce

\begin{tabular}{|c|c|c|c|c|}
\hline Tipo & $\begin{array}{l}\text { Edad de } \\
\text { inicio }\end{array}$ & Clínica & Bioquímica & Actividad BCKAD \% \\
\hline Clásico & Neonatal & $\begin{array}{c}12 \text { horas: } \\
\text { - Olor a Jarabe de arce en cerumen } \\
2-3 \text { días: } \\
\text { • Mala alimentación } \\
\text { • Irritabilidad } \\
4-5 \text { día: } \\
\text { Profundización de encefalopatía } \\
\text { • Opistótono } \\
\text { • "Esgrima", "pedaleo" } \\
\text { • Letargo } \\
\text { • Apneas } \\
7 \text {-10 días: } \\
\text { • Coma } \\
\text { - Insuficiencia respiratoria }\end{array}$ & $\begin{array}{c}\text { 12-24 horas: } \\
\text { - AACR elevados en } \\
\text { plasma } \\
\text { - Aloisoleucina elevada } \\
\text { - AACR elevados en } \\
\text { orina } \\
\text { - DNFH positivo } \\
\text { 2-3 dias: } \\
\text { - Cetonuria }\end{array}$ & $0 \%-2 \%$ \\
\hline Intermedio & Variable & $\begin{array}{l}\text { - Olor a Jarabe de arce en cerumen } \\
\text { - Crecimiento deficiente } \\
\text { - Mala alimentación } \\
\text { • Irritabilidad } \\
\text { - Retrasos del desarrollo } \\
\text { - Encefalopatía en crisis }\end{array}$ & $\begin{array}{l}\text { - Similar al clásico, } \\
\text { menos severo }\end{array}$ & $3 \%-30 \%$ \\
\hline Intermitente & Variable & $\begin{array}{l}\text { - Crecimiento y desarrollo } \\
\text { temprano normal } \\
\text { - Descompensaciones episódicas, } \\
\text { pueden ser graves. }\end{array}$ & $\begin{array}{c}\text { - Alteración del perfil } \\
\text { bioquímico en crisis, } \\
\text { similar al clásico }\end{array}$ & $5 \%-20 \%$ \\
\hline $\begin{array}{c}\text { Sensible a la } \\
\text { tiamina }\end{array}$ & Variable & - Similar al fenotipo intermedio. & $\begin{array}{l}\bullet \text { Mejora de la } \\
\text { tolerancia a la leucina } \\
\text { y perfil bioquímico con } \\
\text { terapia de tiamina }\end{array}$ & $2 \%-40 \%$ \\
\hline
\end{tabular}

Adaptado y modificado de (11).

\section{Diagnóstico}

Como se describe en la literatura, el presente caso es de un recién nacido asintomático, condición que puede persistir desde horas a semanas. La detección ideal se basa en el tamizaje neonatal entre las 24 y 48 horas de vida (9). Durante la crisis, el diagnóstico de confirma a través de: 1) Cuantificación de aminoácidos en plasma a través de cromatografía líquida de aminoácidos en plasma de alto performance (AP-HPLC), lo cual muestra elevaciones de leucina, isoleucina y la valina. 2) Concentración plasmática de aloisoleucina elevada (> $5 \mu \mathrm{mol}$ / L), el cual se considera el metabolito patognomónico (13) y 3) Detección urinaria de alfa-cetoácidos, sin embargo las pruebas cualitativas no permiten la cuantificación de sustancias y pre- 
sentan alta interferencia. Se deben considerar ácidos orgánicos cualitativos en orina, por cromatografía de gases por espectrometría de masas, en donde se evidencia metabolitos patognomónicos como alfa-ceto-isocaproato y alfa-ceto isovalerato.

La prueba de dinitrofenilhidracina (DNFH), es una prueba cualitativa o prueba colorimétrica que presenta interferencia con cetosis de otras causas o ante la presencia de cristales en orina. Actualmente se encuentra en desuso, pero puede ser válida como un tamizaje inicial ante la sospecha clínica. También es positiva en fenilcetonuria, tirosinosis y otros EIM. La cetonuria es un marcador de crisis.

La acidosis láctica, la cetosis y la hiperamonemia leve, no son patognomónicos de la enfermedad pero deben vigilarse junto con los niveles de sodio sérico (7). La medición de actividad de la enzima BCKAD, no es necesaria para el diagnóstico. Debe realizarse solo cuando la sospecha clínica sea fuerte y no se encuentre niveles muy elevados de leucina o sus derivados en sangre. Las pruebas genéticas permiten la confirmación diagnóstica desde el punto de vista molecular (tabla 1).

\section{Tratamiento}

El manejo agudo de los EIM con clínica de intoxicación, tiene como objetivos terapéuticos 1) Suprimir el catabolismo de proteínas endógenas, 2) promover el anabolismo, 3) remover sustancias tóxicas incluido el amonio y 4) prevenir las deficiencias de aminoácidos esenciales (11), manteniendo niveles de leucina $<200 \mu \mathrm{mol} / \mathrm{l}$, de valina entre150 - $250 \mu \mathrm{mol} / \mathrm{l}$ y de isoleucina entre 50 - $150 \mu \mathrm{mol} / \mathrm{l}$. Además de medidas para control de edema cerebral (14). La hidratación debe garantizarse de manera enteral o endovenosa según tolerancia hasta $150 \mathrm{ml} /$ $\mathrm{kg}$. Mantener niveles de sodio entre 138 - $145 \mathrm{mEq} / \mathrm{l}$ (8), garantizando un aporte calórico entre 120 - $130 \mathrm{Cal} / \mathrm{kg} /$ día (7).

En relación a la nutrición, se debe establecer una restricción proteica inicial, entre las 24 a 48 horas de la crisis y después iniciar con un 25 al 50\% de los requerimientos proteicos para la edad (8), excepto en el caso de hiperamonemia. Se recomienda el uso de nutrición parenteral con lípidos, dextrosados, y aminoácidos, libre de AACR, o a través de vía enteral, administrar una fórmula libre de AACR, con cantidades calculadas de isoleucina y valina alcanzando un aporte de $2-3.5 \mathrm{~g} / \mathrm{kg} /$ día de proteína (7). Se reintroduce la proteína intacta o aminoácidos, cuando la leucina plasmática se acerque al límite superior del rango de tratamiento (14).

Las técnicas de depuración extracorpóreas como la hemodiálisis, es el manejo de elección, también puede usarse diálisis peritoneal y hemofiltración según experiencia y disponibilidad. Su indicación se da ante niveles de leucina $>564 \mu \mathrm{mol} / \mathrm{l}$ (33 mg/dl) y síntomas neurológicos graves sin mejoría clínica tras 24 horas con dieta exenta de AACR (7). La Tiamina, se inicia como prueba terapéutica de 1 a 4 semanas a dosis de 50 a 200 $\mathrm{mg} /$ día, y se debe mantener en pacientes respondedores (14). El tratamiento de sostén de la MSUD, consiste en una dieta que limita la cantidad de AACR, a través de una fórmula láctea especial libre de AACR (15). Control de los niveles de leucina, según rango etario y aloisoleucina ausente. En algunos casos es necesario el trasplante hepático, después de un trasplante exitoso, los pacientes pueden consumir una dieta sin restricciones (14).

En el caso presentado, se sospechó un EIM, diagnosticándose MSUD por AOO y confirmándose con AP-HPLC y fenotipo clásico con debut neonatal. Cumpliendo rangos para técnicas de depuración extracorpórea. Se inició diálisis peritoneal y dieta con restricción de AACR, con respuesta favorable evidenciada por mejoría clínica y de laboratorios.

\section{Conclusiones}

Los EIM pueden presentarse con síntomas, signos y laboratorios inespecíficos, requieren considerarse entre los diagnósticos diferenciales en neonatos con encefalopatía o clínica de sepsis, evitando retrasos diagnósticos que conllevan a secuelas neurológicas graves o muerte. El tratamiento debe iniciarse tan pronto como sea posible después del nacimiento, por lo que es imprescindible identificarse en el tamiz metabólico neonatal ampliado y poder realizar diagnósticos presintomáticos y dar tratamiento oportuno evitando descompensaciones y secuelas irreparables. Esta tamización no está incluida en la Ley 1980 de 2019, también conocida como «Ley de tamizaje neonatal de Colombia», por lo que es importante la sospecha clínica y la solicitud de los paraclínicos adecuados. Además, al diagnóstico de EIM siempre se debe realizar consejería genética ante el riesgo de recurrencia en futuros embarazos.

\section{Declaración de conflictos de intereses}

El doctor Adolfo Álvarez ha prestado consultorías como neuropediatra a Orphan, Nutricia, Metabólica, Mitostherapy. Los demás autores declaran no tener conflictos de interés.

\section{REFERENCIAS}

1. Chuang D, Shih V WR. Maple syrup urine disease (branched chain ketoaciduria). Metab Mol Basis Inherit Dis. 2001;(87):12501264.

2. Puffenberger EG. Genetic heritage of the Old Order Mennonites of southeastern Pennsylvania. Am J Med Genet. 2003;121C(1):1831.

3. Couce ML, Castiñeiras DE, Bóveda MD, Baña A, Cocho JA, Iglesias AJ, et al. Evaluation and long-term follow-up of infants with inborn errors of metabolism identified in an expanded screening programme. Mol Genet Metab. 2011;104(4):470-5.

4. Gomez J., Espinosa E., Barrera L. EO. Enfermedad De Orina En Jarabe De Arce : Mejoria Clínica Asociada a Detección Precoz Y Manejo Oportuno. Reporte De Caso y Revisión de Literatura. Revista. 2008;16(49):99-105.

5. Páez P, Suárez F. Urgencias metabólicas y genéticas en la Unidad de Recién Nacidos: Enfermedad de la orina con olor a jarabe de arce. Nutr Hosp. 2015;32(1):420-5.

6. Pettit F, Yeaman S. RL. Purification and characterization of branched chain a-keto acid dehydrogenase complex of bovine kidney. Proc Nati Acad Sci. 1978;75(10):4881-5.

7. Zárate $\mathrm{P}$, Belmont L, Lizárraga $\mathrm{S}$, Muñoz $\mathrm{M}$, Stark A. Metabolopatias en la unidad de cuidados intensivos. Man LA Soc Latinoam Cuid intensivos Pediatr. 2018;(13.3):1-13. 
8. Rodan L, Aldubayan S, Berry G, Levy H. Acute Illness Protocol for Maple Syrup Urine Disease. Pediatr Emer Care. 2017;00(00):1-4.

9. Nellis M, Kasinski A, Carlson M, Allen R, Schaefer AM, Schwartz $\mathrm{EM}$, et al. Relationship of causative genetic mutations in maple syrup urine disease with their clinical expression. Mol Genet Metab. 2003;80:189-95.

10. Gupta D, Bijarnia-Mahay S, Saxena R, Kohli S, Dua-Puri R, Verma J, et al. Identification of mutations, genotype-phenotype correlation and prenatal diagnosis of maple syrup urine disease in Indian patients. Eur J Med Genet. 2015;58(9):471-8.

11. Strauss KA, Puffenberger EG MD. Maple Syrup Urine Disease. GeneReviews $₫$ [Internet]. 2013;1-38.

12. Amartino H, Austral HU, Meritano J. Enfermedad de Orina a Jarabe de Arce. Rev del Hosp Matern Infant Ramón Sardá. 2008;27(002):89-94.
13. Schadewaldt P, Bodner-leidecker A, Hammen H, Wendel U. Significance of L -Alloisoleucine in Plasma for Diagnosis of Maple Syrup Urine Disease. Clin Chem. 1999;45(10):1734-40.

14. Frazier DM, Allgeier C, Homer C, Marriage BJ, Ogata B, Rohr F, et al. Nutrition management guideline for maple syrup urine disease: An evidence- and consensus-based approach. Mol Genet Metab. 2014;112(3):210-7.

15. Strauss KA, Wardley B., Robinson D., Hendrickson C., Rider NL, Puffenberger EG et al. Classical maple syrup urine disease and brain development: Principles of management and formula design. Mol Genet Metab. 2013;99(4):333-45. 